\section{Cyclin encoded by KS herpesvirus}

SIR- Kaposi's sarcoma(KS)associated herpesvirus (KSHV or HHV8) has been strongly implicated in the development of Kaposi's sarcoma and primary effusion lymphomas ${ }^{1,2}$. This herpesvirus was initially described in Kaposi's sarcoma associated with AIDS patients ${ }^{1}$, but has since been found in all epidemiological forms of Kaposi's sarcoma $^{3-5}$. KSHV is a gammaherpesvirus, similar to other oncogenic herpesviruses such as the New World monkey virus, herpesvirus saimiri, and Epstein-Barr virus ${ }^{6}$. Genomic sequencing sequence similarity to genes of Epstein-Barr virus or herpesvirus saimiri which are known to have important oncogenic functions (P. S. M., unpublished data). However, we report here that KSHV contains an open reading frame (ORF 72) with sequence similarity to cellular cyclins, in particular the D-type cyclins $^{7}$ ( $a$ in the figure). (Herpesvirus saimiri also has an open reading frame with similarity to cyclins ${ }^{8,9}$.)

Various lines of evidence suggest that deregulated expression of cellular D-type cyclins is linked to the development of tumours in humans ${ }^{10}$. Cyclin D proteins are KSHV associated diseases. of KSHV has thus far failed to identify

regulatory subunits which activate cellular kinases (CDKs) to phosphorylate checkpoint molecules such as the retinoblastoma-tumour suppressor protein $(\mathrm{RB})^{11}$. The similarity of KSHV ORF 72 to cellular D-type cyclins suggests that its encoded product, KSHV-cyclin, could also stimulate such kinases resulting in their unlicensed activation and, consequently, cause deregulation of cellular proliferation inherent to

To determine whether KSHV-cyclin could activate kinases and perturb control of cell growth in a manner analogous to aberrant expression of D-type cyclins, we engineered an epitope-tagged (c-myc epitope) version of KSHV ORF 72 driven by the cytomegalovirus immediate early promoter. On transfection into COS- 1 cells, this construct gives rise to expression of a polypeptide of relative molecular mass 28,000 that, like the $D$ type cyclins, is localized to the cell nucleus. The KSHV-cyclin protein is associated with kinase activity capable of phosphorylating $\mathrm{RB}$ in vitro at authentic sites, including those previously shown to be a hallmark of RB inactivation ${ }^{12}(b$ in the figure). This supports the hypothesis

a, Amino-acid sequence alignment of the putative 'cyclin box' of KSHV-cyclin (residues 49-82) with HVS cyclin (residues 50-83) and human cyclin D2 (residues 54-87). Identical residues to KSHVcyclin are boxed. $b$, Twodimensional tryptic peptide pattern of full-length RB phosphorylated by KSHV-cyclin immunocomplex in vitro (v-cyc) and of natural RB immunopurified with anti-RB antiserum from ${ }^{32} \mathrm{p}$ labelled human cells (in vivo) demonstrating that authentic sites are phosphory-

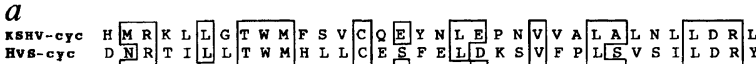

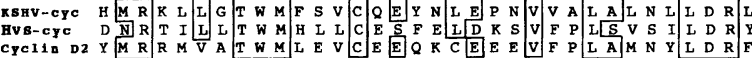

b v-cyc

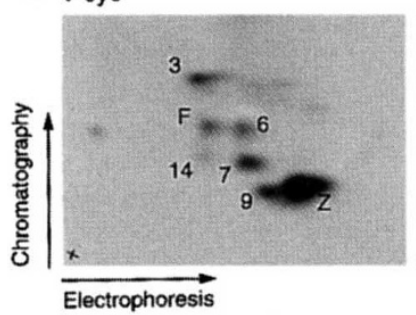
$\overrightarrow{\text { Electrophoresis }}$ lated by the KSHV-cyclin asso ciated kinase ${ }^{12}$. $\quad$, SAOS-2 senescence assay. Photomicrograph of transfected cell monolayers after puromycin selection for 12 days are shown. Monolayers of senescent cells with enlarged cell bodies are seen when cells are transfected with wildtype RB (middle panel) but not in cells transfected with RB 76t mutant

(left panel) or when wild-type RB is transfected together with KSHV-cyclin. Arrowheads in the leftand right-hand panels denote colonies of puromycin resistant, normal size cells which form under the latter two conditions. $d$, Western blot analysis of RB protein in SAOS-2 cell lysates prepared 4 days after transfection showing retarded migration of RB (characteristic of inactive RB) in the KSHV-cyclin transfected sample (RB + RcCMV.V-cyc). Methods (available in full from the authors): SAOS- 2 cells were transfected with expression plasmids encoding a non-functional pRB mutant in which a termination codon was introduced at amino-acid 76 (RB 76t), wild-type RB (RB) or a mixture of wild-type RB and KSHV-cyclin ( $v-c y c)$. Co-transfection with a puromycin resistance plasmid was performed. that KSHV-cyclin associated kinases could mediate the functional inactivation of $\mathrm{RB}$ and thereby overcome the cellcycle arrest imposed by RB.

To test this possibility directly we coexpressed the KSHV-cyclin together with RB in SAOS-2 osteosarcoma cells which, due to the mutation of both RB alleles, lack expression of endogenous $\mathrm{RB}^{13}$. On transfection of wild-type RB, these cells stop growing, resulting in a spreading and senescent-appearing morphology ${ }^{14}$. Coexpression of KSHV-cyclin in these cells abrogates this response, as previously noted with cellular cyclins. Moreover, the RB detected in cell lysates from such cotransfected cells shows a retarded migration characteristic of hyperphosphorylated, inactive $\mathrm{RB}$ ( $c$ in the figure).

Taken together, our findings imply that the ectopic expression of KSHV-cyclin in latently infected host cells can inappropriately activate kinases whose activity normally requires cellular cyclins. This in turn can allow such cells to overcome growth-restriction checkpoints, such as that imposed by RB. Although direct evidence for a transforming role of KSHVcyclin has yet to be established, we have found $\mathrm{KSHV}$-cyclin transcripts in three out of three primary Kaposi's sarcoma biopsies of different aetiology and in a primary effusion lymphoma, indicating a role for KSHV-cyclin in these tumours. The ability of KSHV-cyclin to overcome RBmediated cell cycle arrest in SAOS2 cells provides direct evidence for a mechanism by which this virus could contribute to tumour development.

\section{Yuan Chang \& Patrick S. Moore}

Division of Epidemiology,

School of Public Health and Department of Pathology,

College of Physicians and Surgeons,

Columbia University, New York,

New York 10032, USA

Simon J. Talbot, Chris H. Boshoff, Tamara Zarkowska, Diana Godden-Kent, Hugh Paterson, Robin A. Weiss,

Sibylle Mittnacht*

CRC Centre for Cell and Molecular Biology and Virology Laboratory,

Institute of Cancer Research,

Chester Beatty Laboratories,

London SW3 6JB, UK

* To whom correspondence should be addressed.

1. Chang, Y. et al. Science 266, 1865-1869 (1994).

2. Cesarman, E. et al New Eng. J. Med. 332, 1186-1191 (1995)

3. Boshoff, C. et al. Nature Med. 1, 1274-1278 (1995).

4. Boshoff, C. et al. Lancet 345, 1043-1044 (1995).

5. Moore, P. S. \& Chang, Y. New Eng. J. Med. 332 1181-1185 (1995)

6. Moore, P. S. et al. J. Virol. 70, 549-558 (1996)

7. Cesarman, E., et al. J. Virol. (in the press).

8. Nicholas, J., Cameron, K. R. \& Honess, R. W. Nature 355, 362-365 (1992).

9. Jung, J. U., Stager, M. \& Desrosiers, R. C. Mol. Cell. Biol. 14, 7235-7244 (1994).

10. Jacks, T. \& Weinberg, R. A. Nature 381, 643-644 (1996)

11. Sherr, C.J. Trends Biochem. Sci . 20, 187-190 (1995).

12. Mittnacht, S. et al. EMBO J . 13, 118-127 (1994).

13. Shew, J.Y. et al Oncogene Res. 4, 205-214 (1989).

14. Huang, H.J. et al. Science 242, 1563-1566 (1988). 